\title{
First case of a sutureless Perceval valve delayed proximal migration
}

\author{
Gilles Amr, MD, MSc, ${ }^{a}$ Aly Ghoneim, MD, ${ }^{\mathrm{a}}$ Geneviève Giraldeau, MD, ${ }^{\mathrm{b}}$ and \\ Philippe Demers, MD, MSc, FRCSC ${ }^{a}$, Montréal, Québec, Canada
}

\author{
From the Departments of ${ }^{\mathrm{a} C a r d i a c}$ Surgery and ${ }^{\mathrm{b}}$ Cardiology, Montreal Heart Institute, Montréal, Québec, Canada. \\ Disclosures: Authors have nothing to disclose with regard to commercial support. \\ Received for publication July 26, 2016; revisions received Sept 19, 2016; accepted for publication Sept 21, 2016; \\ available ahead of print Nov 17, 2016. \\ Address for reprints: Philippe Demers, MD, MSc, FRCSC, Department of Cardiac Surgery, Montreal Heart Insti- \\ tute, 5000 Belanger St, Montreal, Quebec H1T 1C8, Canada (E-mail: p.demers@umontreal.ca). \\ J Thorac Cardiovasc Surg 2017;153:e21-3 \\ $0022-5223 / \$ 36.00$ \\ Copyright (C) 2016 by The American Association for Thoracic Surgery \\ http://dx.doi.org/10.1016/j.jtcvs.2016.09.080
}

\section{Video clip is available online.}

An 80-year-old woman presented with severe symptomatic aortic valve stenosis. Her logistic European System for Cardiac Operative Risk Evaluation II was $1.75 \%$ and Society of Thoracic Surgeons score was 2.1\%. Transthoracic echocardiography (TTE) showed preserved left ventricular ejection fraction (LVEF), mean gradient of $52 \mathrm{~mm} \mathrm{Hg}$, aortic valve area (AVA) of $0.62 \mathrm{~cm}^{2}$, and aortic annulus diameter of $22.4 \mathrm{~mm}$.

\section{CLINICAL SUMMARY}

A 5-cm upper ministernotomy was conducted, and cardiopulmonary bypass (CPB) was initiated using standard aorto-atrial cannulation. The technique of sutureless valve implantation has been described ${ }^{1,2}$ and is summarized briefly in the current report. After a transverse aortotomy, the 3 native stenosed cusps were removed, annular decalcification was carried out, and a medium-sized Perceval S (Sorin Group, Saluggia, Italy) prosthesis was implanted. The CPB and crossclamp times were 59 and 39 minutes, respectively. The intraoperative transesophageal echocardiography control revealed a well-functioning and properly positioned prosthesis. The postoperative course was noteworthy for the apparition of a total atrioventricular block requiring a permanent pacemaker implantation. At discharge, TTE showed a trace of paravalvular leakage (PVL), a $1.5 \mathrm{~cm}^{2}$ AVA, and a mean gradient of $17 \mathrm{~mm} \mathrm{Hg}$ (Figure 1, $A$ and $B$ ). The patient missed her follow-up examination after surgery and was readmitted 15 months later with congestive heart failure. The

\section{DISCUSSION}

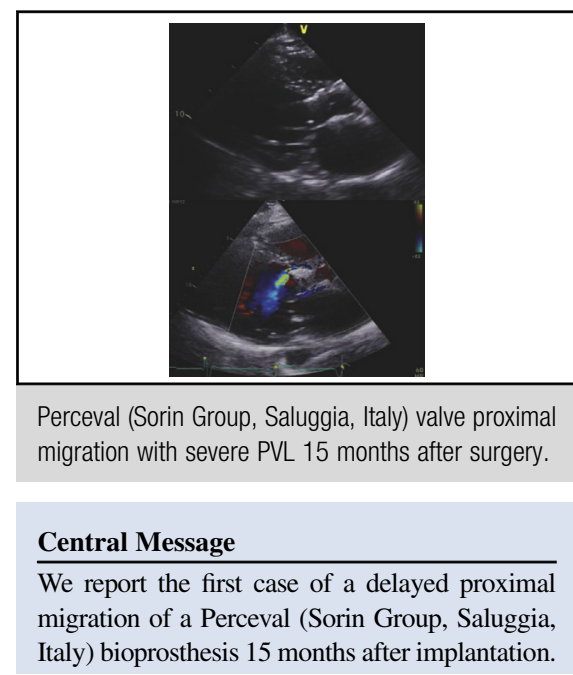

See Editorial Commentary page e25.

TTE showed a $45 \% \mathrm{LVEF}$, an aortic mean gradient of $15.5 \mathrm{~mm} \mathrm{Hg}$, and severe PVL caused by a distorted and displaced valve (Video 1 and Figure $1, C$ and $D$ ). The computed tomography scan confirmed the proximal migration of the prosthesis (Figure 2, $A-C$ ). Reoperation was performed using a median sternotomy. Concordant with the preoperative imaging, we identified a proximal migration of the valve into the left ventricular outflow tract in the right coronary sinus (Figure $2, D$ ). The valve was easily explanted and showed no signs of infection or deterioration (Figure 2, $E$ and $F$ ) after careful inspection. Of note, appropriate Perceval sizing after complete calcium debridement was 1 size larger than at the first operation. Finally, standard aortic valve replacement was performed using a 23mm Trifecta prosthesis (St Jude Medical, Inc, St Paul, Minn). The control TTE showed satisfactory valve function (8.6 $\mathrm{mm} \mathrm{Hg}$ mean gradient and $1.8 \mathrm{~cm}^{2}$ AVA), no PVL, and a slight improvement in the LVEF. The postoperative course was uneventful.

Sutureless valves are gaining momentum as an alternative to standard surgical replacement. ${ }^{1}$ This new technique provides shorter crossclamp and CPB times, especially with concomitant procedures, and easy implantation in 


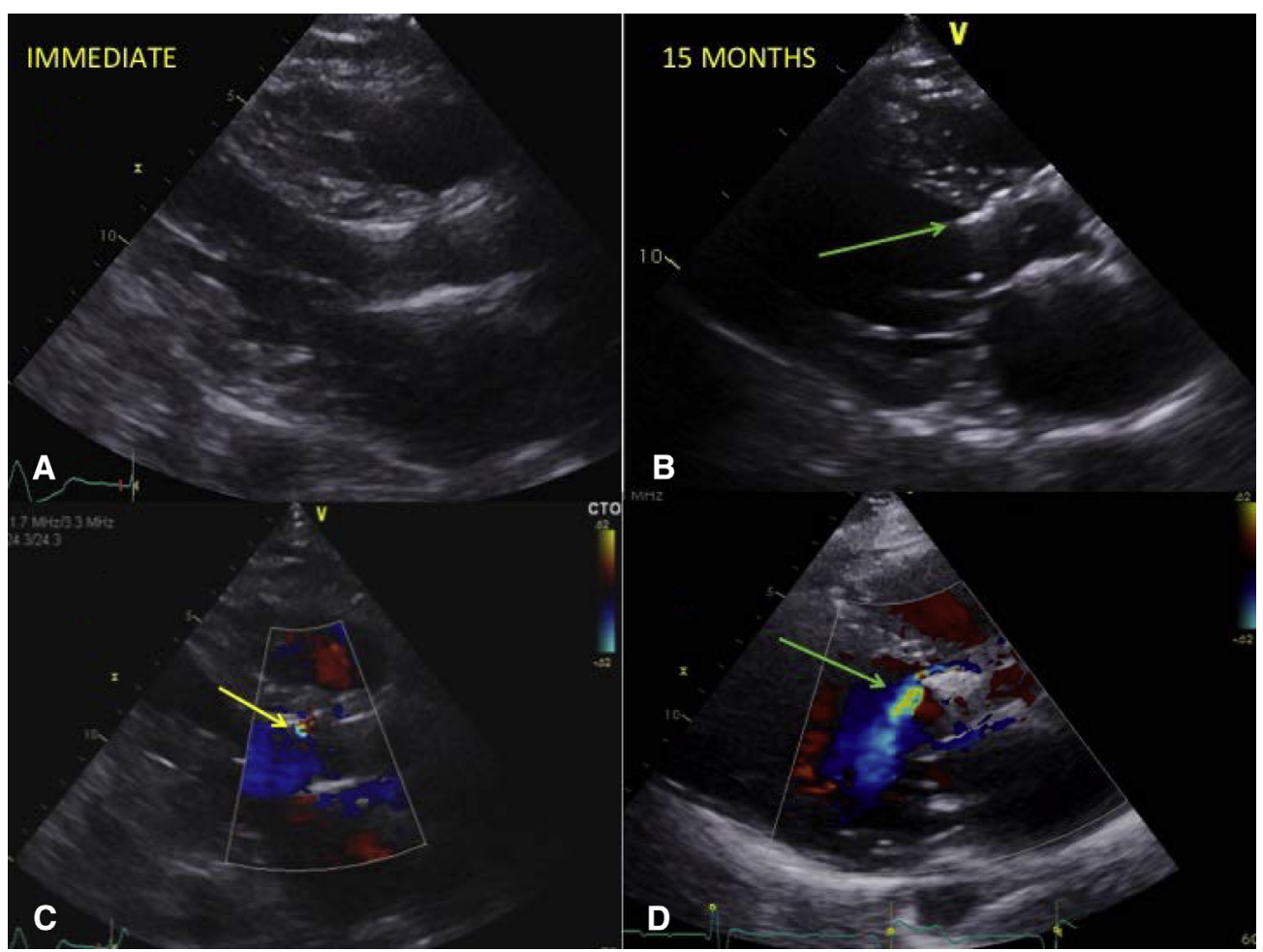

FIGURE 1. A and B, Postoperative TTE control showing a normal positioning of the Perceval (Sorin Group, Saluggia, Italy) valve with a trace of PVL: yellow arrow. C and D, Readmission TTE revealing the Perceval valve's dislodgement into the left ventricular outflow tract with severe leakage: green arrows.

minimally invasive procedures. Our experience with the Perceval valve started in 2011, and approximately 300 valves were implanted in patients before this case. Our early results and complications have been reported. ${ }^{2}$ We noted $11 \%$ mild and $0 \%$ moderate or severe PVL, $17 \%$

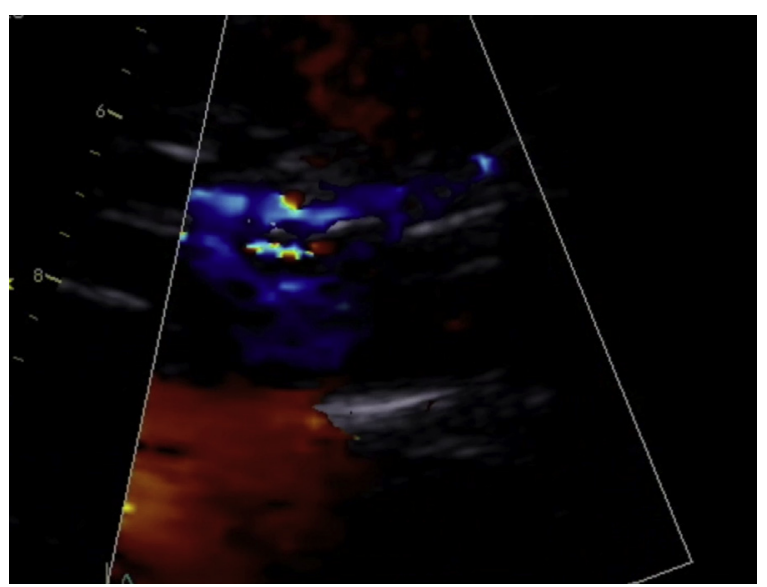

VIDEO 1. The first echocardiography clips show the satisfactory position of the Perceval (Sorin Group, Saluggia, Italy) valve with a trace of PVL in long-axis and short-axis views after the first surgery. The second echocardiography clips reveal the delayed proximal migration and the severe PVL in long-axis, 3-chamber, and short-axis views. Video available at: http:// www.jtcvsonline.org/article/S0022-5223(16)31421-0/addons. pacemaker implantation, and a case of structural valve deterioration. ${ }^{3}$ To our knowledge, this is the first reported case of a Perceval valve late proximal migration. Intraoperative Perceval malposition is rare and usually evident on the intraoperative transesophageal echocardiography, allowing prompt correction. One case of a sutureless valve late migration ( 3 months after surgery) has been described concerning a 3F Enable valve (ATS, Medtronic, Inc, Minneapolis, Minn), and although the valve was attached by a stitch, it migrated upward into the ascending aorta. ${ }^{4}$ The Perceval valve, like transcatheter aortic valves, is truly sutureless and relies on the stent radial forces. This delayed unanticipated migration after an accurate positioning is concerning. Several hypotheses could explain the delayed migration, including the inadequate sizing or the nonuniformly decalcified annulus. In our case, we believe that the dislodgement could be related to valve undersizing, probably caused by a heterogeneous debridement. A similar event was mentioned in the literature concerning a balloon-expandable transcatheter valve, in which case, besides a mild aortic regurgitation, the prosthesis looked stable at the predischarge TTE, and the patient was admitted 43 days later for late migration. ${ }^{5}$ This report emphasizes the importance of systematic aortic annulus decalcification before meticulous sizing to obtain durable outcomes. 

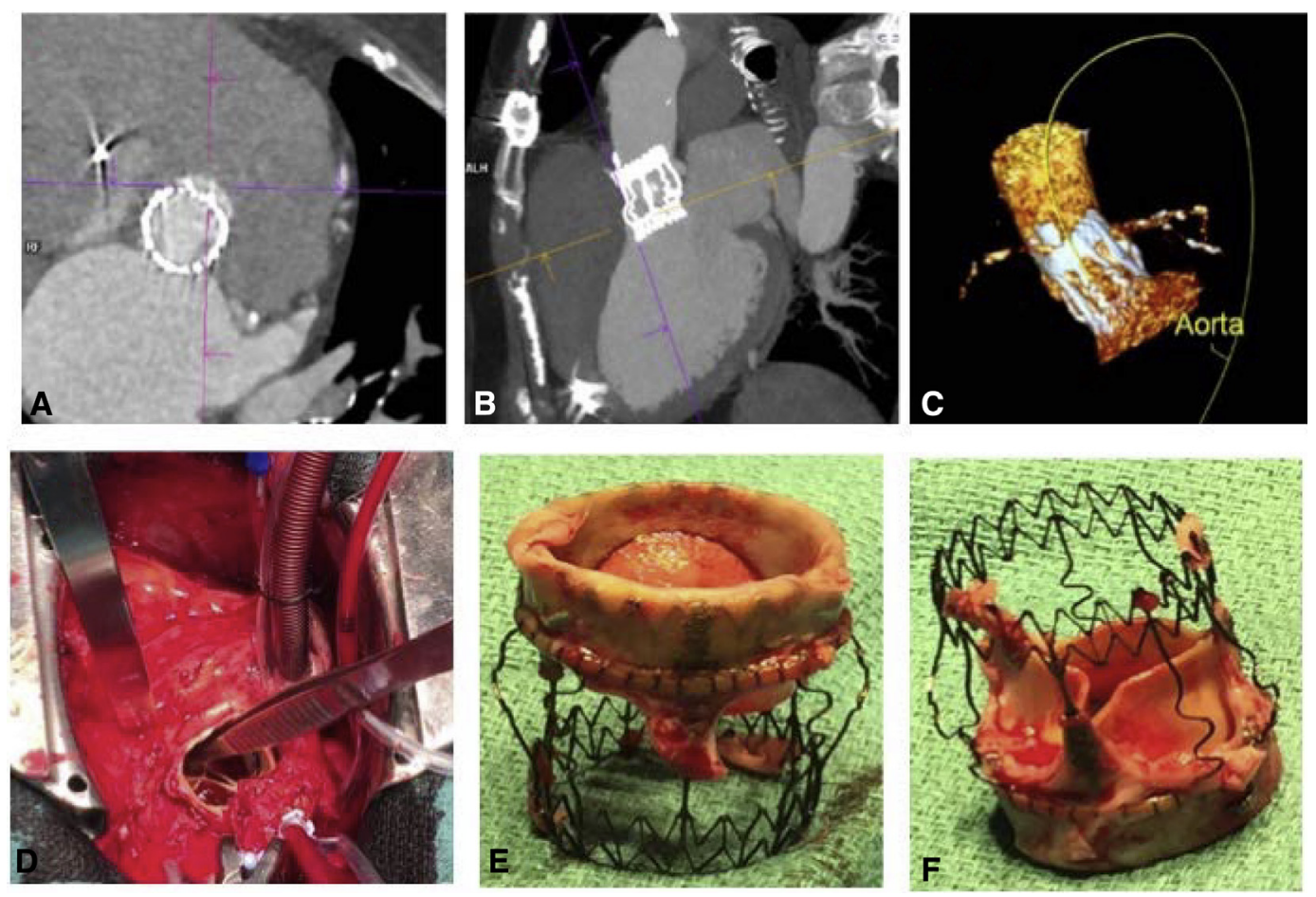

FIGURE 2. A, Axial computed tomography scan section revealing PVL. B, Sagittal computed tomography scan section showing a distorted valve migrating into the left ventricular outflow tract. C, Three-dimensional reconstruction showing the valve's position relative to the aorta's axis. D, Intraoperative surgical view with the DeBakey forceps indicating the dislodgment at the level of the right coronary cusp. E and F, The explanted prosthesis with no signs of deterioration or infection.

\section{References}

1. Shrestha M, Fischlein T, Meuris B, Flameng W, Carrel T, Madonna F, et al. European multicenter experience with the sutureless Perceval valve: clinical and haemodynamic outcomes up to 5 years in over 700 patients. Eur J Cardiothoracic Surg. 2016;49:234-41.

2. Mazine A, Teoh K, Bouhout I, Bhatnagar G, Pelletier M, Voisine P, et al. Sutureless aortic valve replacement: a Canadian multicenter study. Can J Cardiol. 2015; $31: 63-8$.
3. Bouhout I, Noly PE, Parisi A, Bouchard D. First case of Perceval S prosthesis early structural valve deterioration: not an easy reoperation. J Thorac Cardiovasc Surg. 2016;152:e71-3.

4. Concistrè G, Miceli A, Chiaramonti F, Glauber M. Delayed dislocation of a sutureless aortic bioprosthesis: the first case. Interact Cardiovasc Thorac Surg. 2012;14:892-3.

5. Pang PY, Chiam PT, Chua YL, Sin YK. A survivor of late prosthesis migration and rotation following percutaneous transcatheter aortic valve implantation. Eur J Cardiothorac Surg. 2012;45:1195-6. 